\title{
AVALIAÇÃO VESTIBULAR EM MULHERES COM DISFUNÇÃO TEMPOROMANDIBULAR
}

\section{Vestibular evaluation in women with temporomandibular dysfunction}

\author{
Bianca Simone Zeigelboim ${ }^{(1)}$, Ari Leon Jurkiewicz ${ }^{(2)}$, \\ Jackeline Martins-Bassetto ${ }^{(3)}$, Karlin Fabianne Klagenberg ${ }^{(4)}$
}

\begin{abstract}
RESUMO
Objetivo: avaliar o comportamento vestibular em pacientes com disfunção temporomandibular. Métodos: avaliaram-se 27 pacientes do sexo feminino, na faixa etária de 30 a 53 anos, encaminhadas do Centro de Diagnóstico e Tratamento da Articulação Temporomandibular para o Laboratório de Otoneurologia da Universidade Tuiuti do Paraná. Realizaram-se os seguintes procedimentos: anamnese, inspeção otológica e avaliação vestibular por meio da vectoeletronistagmografia. Resultados: as queixas mais freqüentes foram: dificuldade ou dor ao movimento do pescoço $(77,7 \%)$, dor irradiada para ombro/braço $(77,7 \%)$, zumbido e formigamento de extremidade superior $(77,7 \%)$, tontura e dor de cabeça $(66,6 \%)$, ansiedade $(55,5 \%)$, sensação de cabeça oca $(51,8 \%)$, agitação durante o sono $(51,8 \%)$ e depressão $(51,8 \%)$. O exame vestibular esteve alterado em 20 pacientes $(74,0 \%)$ na prova calórica. Houve freqüência de alteração no sistema vestibular periférico. Houve predomínio das síndromes vestibulares periféricas deficitárias. Conclusão: ressalta-se a importância de se estudar a relação do sistema vestibular com a disfunção temporomandibular uma vez que observamos, na presente pesquisa, um número elevado de alteração no exame labiríntico.
\end{abstract}

DESCRITORES: Articulação Temporomandibular; Tontura; Eletronistagmografia; Vertigem; Doenças do Labirinto

\section{INTRODUÇÃO}

A disfunção temporomandibular (DTM) foi descrita por James Costen em 1934 como sendo uma síndrome constituída de sintomas heterogêneos e de pertinência odontológica e otoneurológica, tendo como causa uma disfunção da articulação temporomandibular (ATM). Esta síndrome passou a ser reconhecida como Costen's Syndrome ${ }^{1}$. O autor referiu que a perda de suporte dental posterior e as queixas otológicas, com origem em problemas

(1) Fonoaudióloga; Coordenadora do Programa de Mestrado e Doutorado em Distúrbios da Comunicação da Universidade Tuiuti do Paraná; Doutora em Ciências dos Distúrbios da Comunicação Humana pela Universidade Federal de São Paulo.

(2) Médico Clínico; Professor Adjunto do Programa de Mestrado e Doutorado em Distúrbios da Comunicação da Universidade Tuiuti do Paraná; Doutor em Anatomia pela Universidade Federal de São Paulo.

(3) Fonoaudióloga da Empresa de Aparelhos Audiotec; Mestre em Distúrbios da Comunicação pela Universidade Tuiuti do Paraná.

(4) Fonoaudióloga da Clínica Batel; Mestre em Distúrbios da Comunicação pela Universidade Tuiuti do Paraná. oclusais, poderiam ser provocadas por compressão da tuba auditiva, pressão do nervo auriculotemporal e/ou pressão do nervo da corda do tímpano ${ }^{1}$.

Os distúrbios da articulação temporomandibular são um "conjunto de sinais e sintomas relacionados a desordens funcionais e estruturais do sistema estomatognático" ${ }^{2}$.

Os indivíduos com DTM podem apresentar deslocamentos espontâneos da mandíbula, traumas nas articulações temporomandibulares, hipomobilidade crônica da mandíbula e síndrome da disfunção miofacial temporomandibular, além de sintomas otoneurológicos como otalgia, zumbido, vertigem, tontura, hipoacusia, entre outros ${ }^{3}$. Esses sintomas foram correlacionados com possíveis causas de DTM por diversos autores ${ }^{4-10}$.

Existe uma conexão funcional entre o aparelho mastigatório e a orelha. Espasmos na musculatura mastigatória causam contração reflexa dos músculos tensor do tímpano e tensor do véu palatino, podendo ocorrer, assim, um desequilíbrio de tensão na cadeia ossicular. Como conseqüência, poderá haver um aumento da impedância das estruturas condutoras do som com diminuição da transmissão sonora ${ }^{4}$. 
Diversos estudos ${ }^{3,4-10}$ correlacionam os sinais e sintomas otoneurológicos com a disfunção da ATM devido a proximidade anatômica e estrutural. Apesar desta relação existir, a patogênese da mesma é desconhecida. Autores ${ }^{11}$ hipotetizam esta correlação baseada em três teorias. A primeira, na possibilidade de transmissão mecânica de força da ATM à oreIha média, através do ligamento discomaleolar; a segunda, da possível irritação direta pelo côndilo da mandíbula do nervo auriculotemporal e a mais recente, a da hipertonicidade dos músculos tensores do tímpano e do véu palatino, baseada na inervação comum trigeminal destes músculos e dos músculos mastigatórios inseridos na mandíbula.

O objetivo do presente estudo foi avaliar o comportamento vestibular em pacientes portadores de DTM.

\section{MÉTODOS}

Avaliaram-se 27 pacientes do sexo feminino com diagnóstico de disfunção temporomandibular, na faixa etária de 30 a 53 anos, encaminhadas do Centro de Diagnóstico e Tratamento da Articulação Temporomandibular para o Laboratório de Otoneurologia da Universidade Tuiuti do Paraná.

Foram excluídas da pesquisa pacientes que apresentaram comprometimentos psicológicos, visuais, musculoesqueléticos importantes e outros distúrbios que impossibilitassem a realização da avaliação vestibular.

Realizaram-se os seguintes procedimentos:

\section{Anamnese}

Aplicou-se um questionário com ênfase aos sinais e sintomas otoneurológicos, antecedentes pessoais e familiares.

\section{Avaliação Otorrinolaringológica}

Realizada com o objetivo de excluir qualquer alteração que pudesse interferir no exame.

\section{Avaliação Vestibular}

Os pacientes foram submetidos às seguintes provas que compõem o exame vestibular:

\section{-Sem registro}

" Pesquisou-se o nistagmo e a vertigem de posição/ posicionamento através da manobra de Brandte Daroff ${ }^{12}$.

. Pesquisaram-se os nistagmos espontâneo e semi-espontâneo com os olhos abertos, no olhar frontal e a $30^{\circ}$ de desvio do olhar para a direita, esquerda, para cima e para baixo.

\section{-Com registro}

Para a realização da vectoeletronistagmografia (VENG) utilizou-se um aparelho termossensível, com três canais de registro, da marca Berger, modelo VN316. Após a limpeza da pele das regiões periorbitárias com álcool, colocaram-se, fixados com pasta eletrolítica, um eletródio ativo no ângulo lateral de cada olho e na linha média frontal, formando um triângulo isósceles, que permitiu a identificação dos movimentos oculares horizontais, verticais e oblíquos. Este tipo de VENG possibilitou obter medidas mais precisas da velocidade da componente lenta (correção vestibular) do nistagmo.

Utilizou-se uma cadeira rotatória pendular decrescente da marca Ferrante, de um estimulador visual marca Neurograff, modelo EV VEC, e de um otocalorímetro a ar, da marca Neurograff, modelo NGR 05.

Realizaram-se as seguintes provas oculares e labirínticas à VENG, segundo os critérios propostos por diversos autores ${ }^{13,14}$.

"Calibração dos movimentos oculares: nesta etapa do exame, o aspecto clínico avaliado foi a regularidade do traçado, tornando as pesquisas comparáveis entre si.

"Pesquisa dos nistagmos espontâneo (olhos abertos e fechados) e semi-espontâneo (olhos abertos): neste registro avaliaram-se a ocorrência, direção, efeito inibidor da fixação ocular (EIFO) e o valor da velocidade angular da componente lenta (VACL) máxima do nistagmo.

" Pesquisa do rastreio pendular para a avaliação da ocorrência e do tipo de curva.

"Pesquisa do nistagmo optocinético, à velocidade de $60^{\circ}$ por segundo, nos sentidos anti-horário e horário, na direção horizontal. Avaliaram-se a ocorrência, direção, VACL máxima às movimentações anti-horária e horária do nistagmo.

" Pesquisa dos nistagmos pré e pós-rotatórios à prova rotatória pendular decrescente, com estimulação dos ductos semicirculares laterais, anteriores e posteriores. Para a estimulação dos ductos semicirculares laterais (horizontais) a cabeça foi fletida $30^{\circ}$ para frente. Na etapa seguinte, para a sensibilização dos ductos semicirculares anteriores e posteriores (verticais) o posicionamento da cabeça foi de $60^{\circ}$ para trás e $45^{\circ}$ à direita e, a seguir, 60 para trás e $45^{\circ}$ à esquerda, respectivamente. Observou-se a ocorrência, direção, freqüência às rotações antihorária e horária do nistagmo.

"Pesquisa dos nistagmos pré e pós-calóricos realizada com o paciente posicionado de forma que a cabeça e o tronco estivessem inclinados $60^{\circ}$ para trás, para estimulação adequada dos ductos semicirculares laterais. O tempo de irrigação de cada oreIha com ar a $42^{\circ} \mathrm{C}, 20^{\circ} \mathrm{C} \mathrm{e} 10^{\circ} \mathrm{C}$ durou 80 s para cada temperatura e as respostas foram registradas com 
os olhos fechados e, a seguir, com os olhos abertos para a observação do EIFO. Nesta avaliação verificaram-se a direção, os valores absolutos da VACL e o cálculo das relações da preponderância direcional e predomínio labiríntico do nistagmo pós-calórico.

Os exames foram realizados por fonoaudiólogas pertencentes ao núcleo de pesquisa "avaliação otoneurológica no diagnóstico diferencial das vestibulopatias" do laboratório de otoneurologia da UTP.

A pesquisa foi aprovada pelo Comitê de Ética Institucional sob número 035/2004 e os pacientes assinaram o Termo de Consentimento Livre e Esclarecido.

Aplicou-se o teste de Diferença de Proporções com a finalidade de comparar os resultados da prova calórica (analisando os valores absolutos e relativos) e do resultado do exame vestibular. Fixou-se 0,05 ou $5 \%$ o nível de rejeição na hipótese de nulidade.

\section{RESULTADOS}

A freqüência dos sinais e sintomas otoneurológicos está exposta na Tabela 1. Outros achados clínicos em pacientes com DTM estão descritos na Tabela 2. Evidencia-se que as manifestações clínicas mais observadas foram: ansiedade $(55,5 \%)$, agitação durante o sono $(51,8 \%)$, depressão $(51,85)$, insônia $(48,1 \%)$ e fadiga $(44,4 \%)$.

As pesquisas do nistagmo de posição, calibração dos movimentos oculares, nistagmos espontâneo e semi-espontâneo, rastreio pendular e nistagmos optocinético e per-rotatório não evidenciaram alterações. As alterações observadas ocorreram apenas na prova calórica.

À prova calórica, ocorreram sete casos $(26,0 \%)$ de normorreflexia e 20 casos $(74,0 \%)$ de alterados. Destes, seis casos $(22,2 \%)$ de preponderância direcional do nistagmo (PDN) assimétrica, cinco casos $(18,5 \%)$ de hiporreflexia labiríntica unilateral, quatro casos $(14,8 \%)$ de hiporreflexia labiríntica bilateral, três casos $(11,1 \%)$ de hiperreflexia labiríntica unilateral, dois casos $(7,4 \%)$ de preponderância labiríntica $(\mathrm{PL})$ assimétrica e sete casos $(26,0 \%)$ de normorreflexia, conforme Figura 1.

À prova calórica analisando os valores absolutos e relativos ocorreram de acordo com o teste de Dife- rença de Proporções, houve diferença significativa na análise da prova calórica.

Em 20 casos (74,0\%) ocorreram síndromes vestibulares periféricas, sendo seis casos $(22,2 \%)$ de síndrome vestibular periférica irritativa, cinco casos $(18,5 \%)$ de síndrome vestibular periférica deficitária à esquerda, quatro casos $(14,8 \%)$ de síndrome vestibular periférica deficitária bilateral, três casos $(11,1 \%)$ de síndrome vestibular periférica irritativa à direita $e$ dois casos $(7,4 \%)$ de síndrome vestibular periférica deficitária à direita. Em sete casos $(26,0 \%)$ o exame foi normal, conforme Tabela 3.

De acordo com o teste de Diferença de Proporções, houve diferença significativa na análise do resultado do exame vestibular.

Tabela 1 - Freqüência dos sinais e sintomas otoneurológicos em pacientes com disfunção temporomandibular

\begin{tabular}{|c|c|c|}
\hline SINAIS E SINTOMAS OTONEUROLÓGICOS & $\mathbf{N}^{\circ}$ & FREQÜÊNCIA \% \\
\hline Dificuldade ou dor ao movimento do pescoço & 21 & 77,7 \\
\hline Dor irradiada para ombro ou braço & 21 & 77,7 \\
\hline Zumbido & 21 & 77,7 \\
\hline Formigamento de extremidade superior & 21 & 77,7 \\
\hline Tontura & 18 & 66,6 \\
\hline Dor de cabeça & 18 & 66,6 \\
\hline Sensação de cabeça oca & 14 & 51,8 \\
\hline Escurecimento de visão & 13 & 48,1 \\
\hline Estalo no pescoço & 13 & 48,1 \\
\hline Desequilíbrio à marcha & 12 & 44,4 \\
\hline Sudorese & 10 & 37,0 \\
\hline Náusea & 10 & 37,0 \\
\hline Pressão na cabeça & 9 & 33,3 \\
\hline Dificuldade para engolir & 8 & 29,6 \\
\hline Visão dupla & 6 & 22,2 \\
\hline Visão borrada & 6 & 22,2 \\
\hline Paralisia facial & 4 & 14,8 \\
\hline Formigamento da face & 4 & 14,8 \\
\hline Palidez & 4 & 14,8 \\
\hline Dificuldade para falar & 4 & 14,8 \\
\hline Sensação de flutuação & 3 & 11,1 \\
\hline Sensação de oscilação & 2 & 7,4 \\
\hline Sensação que vai desmaiar & 2 & 7,4 \\
\hline Incoordenação do movimento & 1 & 3,7 \\
\hline Vômito & 1 & 3,7 \\
\hline Tremor & 1 & 3,7 \\
\hline Sensação de instabilidade & 1 & 3,7 \\
\hline
\end{tabular}


Tabela 2 - Outras manifestações clínicas em pacientes com disfunção temporomandibular

\begin{tabular}{lcc}
\hline \multicolumn{1}{c}{ OUTRAS MANIFESTAÇÕES CLÍNICAS } & N $^{\circ}$ & FREQÜÊNCIA \% \\
\hline Ansiedade & 15 & 55,5 \\
Agitação durante o sono & 14 & 51,8 \\
Depressão & 14 & 51,8 \\
Insônia & 13 & 48,1 \\
Fadiga & 12 & 44,4 \\
Palpitação & 8 & 29,6 \\
Alteração da voz & 7 & 25,9 \\
Medo & 7 & 25,9 \\
Pesadelo & 7 & 25,9 \\
Alteração do paladar & 6 & 22,2 \\
Convulsão & 4 & 14,8 \\
Alteração do olfato & 2 & 7,4 \\
Queda & 2 & 7,4 \\
Cegueira temporária & 1 & 3,7 \\
Perda de consciência & 1 & 3,7 \\
Alucinação & 1 & 3,7 \\
\hline
\end{tabular}

№ - número de pacientes

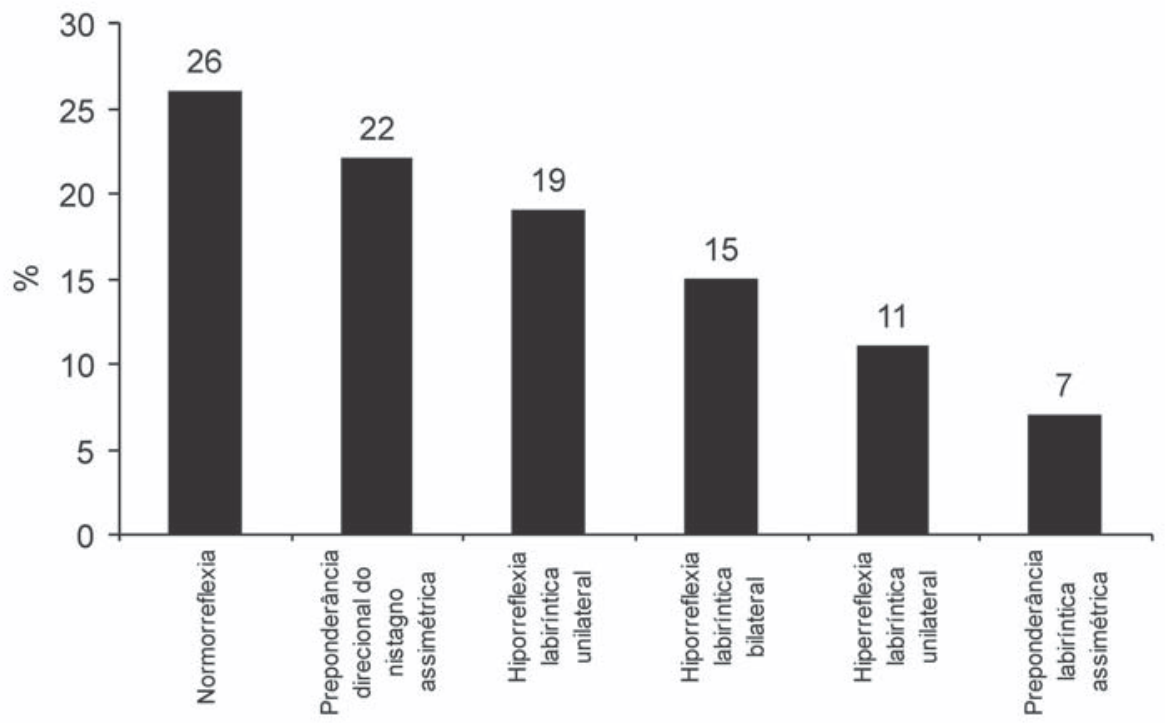

O teste de Diferença de Proporções resultou em $p=0,0337$ e ao nível de significância $\alpha=0,05$ (5\%), a diferença é significativa, pois $p<\alpha$

Figura 1 - Distribuição das freqüências relativas e absolutas dos resultados obtidos na prova calórica, em pacientes com disfunção temporomandibular 
Tabela 3 - Resultado do exame vestibular em pacientes com disfunção temporomandibular

\begin{tabular}{lcc}
\multicolumn{1}{c}{ ACHADOS } & No $^{\circ}$ & FREQÜÊNCIA \% \\
\hline Exame Vestibular Normal & 7 & 25,9 \\
Síndrome Vestibular Periférica & 6 & 22,2 \\
$\quad$ Irritativa & 5 & 18,5 \\
Síndrome vestibular periférica & & \\
$\quad$ deficitária à esquerda & 4 & 14,8 \\
Síndrome Vestibular Periférica & & 11,1 \\
$\quad$ Deficitária Bilateral & 3 & 7,4 \\
Síndrome Vestibular Periférica & & \\
$\quad$ Irritativa á Direita & 2 & \\
Síndrome Vestibular Periférica & & \\
$\quad$ Deficitária à Direita &
\end{tabular}

Legenda: $\mathrm{N}^{\circ}$ - número de pacientes

O teste de Diferença de Proporções resultou em $p=0,0337$ e ao nível de significância $\alpha=0,05(5 \%)$, a diferença é significativa, pois $p<\alpha$.

\section{DISCUSSÃO}

A correlação entre os sinais e sintomas otoneurológicos e a disfunção da ATM é hipotetizada em diversos estudos ${ }^{3,4-10}$. A freqüência dos sintomas referidos pelos pacientes no presente estudo foram: dificuldade ou dor ao movimento do pescoço, dor irradiada para ombro/braço, zumbido e formigamento de extremidade superior $(77,7 \%)$, tontura e dor de cabeça $(66,6 \%)$, ansiedade $(55,5 \%)$, sensação de cabeça oca, agitação durante o sono e depressão $(51,8 \%)$, valores percentuais para cada queixa. Estas manifestações estiveram em concordância com diversos estudos ${ }^{5,9-10,15-22}$ que citaram o zumbido como sintoma prevalente. Outros sintomas prevalentes observados na literatura ${ }^{10,15,19,20}$ foram: otalgia, perda auditiva, cefaléia, sensação de cabeça oca, escurecimento da visão e tontura não rotatória. Sintomas como ansiedade, depressão, insônia, agitação durante o sono, medo e irritabilidade, verificados neste estudo, foram igualmente referidos por autores ${ }^{18,21}$ que observaram que os mesmos poderiam se relacionar a problemas de ordem psicológica, classificando-os como sintomas acessórios otoneurológicos.

Estudo ${ }^{4}$ refere uma conexão funcional entre o aparelho mastigatório e a orelha. Filogeneticamente, os ossículos da orelha média são interpretados como ossos da mandíbula, que passaram a serem utilizados para ouvir, e os músculos tensores do véu palatino e do tímpano, como músculos mastigatórios. Espasmos na musculatura mastigatória causam contração reflexa dos músculos tensor do tímpano e tensor do véu palatino, ocorrendo, assim, um desequilíbrio de tensão na cadeia ossicular. Como conseqüência, poderá haver um aumento da impedância ou resistência das estruturas condutoras do som com diminuição da transmissão sonora. Os músculos tensores do véu palatino e do tímpano dividem com os músculos mastigatórios, envolvidos na DTM, proximidade anatômica e funcional, além da mesma inervação pelo trigêmeo. Assim, esses espasmos prejudicam a abertura da tuba auditiva o que ocasiona pressão nas oreIhas (plenitude auricular), desequilíbrio e perda de audição. Quanto aos espasmos do músculo tensor do tímpano, estes provocam os mesmos sintomas, além de otalgia, zumbido e cefaléia. Sendo assim, o autor ${ }^{4}$ associou a diminuição dos sintomas auditivos com o restabelecimento da oclusão da ATM.

Autore ${ }^{16}$ buscaram determinar se o zumbido e a vertigem eram sintomas normalmente mais prevalentes em pacientes com DTM. Os resultados confirmaram o que vários autores já mencionados têm 
notado que existe uma relação entre DTM e sintomas otoneurológicos. Apesar dessa relação existir, a patogênese da mesma é desconhecida. A otalgia existente em alguns pacientes com DTM pode ser explicada pela proximidade anatômica das estruturas da orelha e da ATM. A relação causal não pôde ser afirmada nesta associação, DTM e sintomas de zumbido e vertigem, mas os autores deixam clara a necessidade de que haja uma maior investigação dessa associação.

Autores ${ }^{19}$ investigaram uma amostra de 36 pacientes com DTM através de exames otoneurológicos e notaram, em todos os casos de síndrome de DTM, algum tipo de sintomatologia otoneurológica: $28 \mathrm{ca}$ sos $(77 \%)$ com vertigem, tontura não rotatória, cefaléia, sensação de desligamento e escurecimento de visão ou sensação de "cabeça oca"; em nove casos (25\%), hipoacusia e hipersensibilidade a sons intensos e zumbidos, entre outros sintomas. Os pesquisadores verificaram discretas alterações audiométricas e impedanciométricas, entretanto, os achados vestibulares foram bastante relevantes nos pacientes que apresentaram sintomas auditivos e/ ou vestibulares (36 casos). Os autores referem que não foi possível estabelecer relações do tipo causaefeito entre DTM e a presença de sinais e sintomas otoneurológicos, mas que devido ao número importante de achados justificaria a inclusão sistemática da investigação otoneurológica na avaliação clínica da afecção.

Pesquisadores ${ }^{11}$ realizaram uma análise sobre sintomas otovestibulares presentes na disfunção da ATM. Os mesmos selecionaram um grupo de 53 pacientes adultos e em tratamento para DTM. Foram coletados dados iniciais relativos à freqüência dos sintomas otovestibulares e cada paciente passou por avaliação eletromiográfica computadorizada. Para análise dos dados os pacientes foram divididos em dois grupos: um grupo que não apresentava nenhum sintoma otológico, e outro com pelo menos um sintoma otológico. Foram comparados os valores da eletromiografia para cada músculo avaliado. Os resultados foram: $67,9 \%$ dos examinados apresentaram pelo menos um sintoma otológico, sendo os mais freqüentes o de ouvir menos $(41,5 \%)$, seguido por otalgia e vertigem $(7,5 \%)$ em cada.

Autore ${ }^{22}$ compararam as características do zumbido nos pacientes portadores de DTM e em pacientes com afecções otoneurológicas. Foram pesquisados 60 pacientes divididos em dois grupos de 30 pacientes (um de DTM e outro com afecção otoneurológica). Quanto aos resultados houve diferença entre os dois grupos em relação à intensidade do zumbido, queixa principal, e os resultados audiológicos. O zumbido do grupo DTM foi de alta freqüência, de aparecimento esporádico, como um apito de intensidade moderada e sem interferência nas atividades de vida diária. O zumbido do grupo de afecção otoneurológica foi predominantemente agudo, variando de intenso a muito intenso, sendo contínuo ou pulsátil, podendo apresentar características de sons complexos e não comparáveis a tons puros, prejudicando as atividades de vida diária e o sono dos pacientes. Os sintomas de otalgia e plenitude auricular prevaleceram no grupo DTM, e as queixas de zumbido e diminuição da audição e discriminação auditiva no grupo de afecção otoneurológica. Essas diferenças fornecem índices para caracterizar cada grupo auxiliando no diagnóstico diferencial, porém, não devem ser os únicos parâmetros, pois existem doenças auditivas coexistindo com a DTM, sem relação causa-efeito.

Segundo os autores ${ }^{23}$ os sintomas freqüentemente associados com DTM são cefaléia, zumbido, otalgia, percepção de ruído da ATM e alteração de equilíbrio, além de problemas de maloclusão e palpação dolorosa de estruturas temporomandibulares. Em alguns movimentos o côndilo mandibular pode exercer pressão no nervo auriculotemporal, próximo à cápsula da ATM, agindo como um gatilho para desencadear o processo doloroso na região temporal. Citaram que a DTM ocorreu mais freqüentemente na quarta década de vida e no sexo feminino. Segundo os autores, a mandíbula e os ossículos da orelha média têm a mesma origem embriológica, esclarecendo o motivo de várias malformações da orelha média estarem associadas com alterações mandibulares. A anatomia e a biomecânica da ATM inter-relacionam-se, por estarem intimamente relacionadas com estruturas e funções aurais.

A freqüência do sexo feminino também foi observada por autores ${ }^{24}$ que estudaram 75 pacientes portadores de DTM. Os autores verificaram também a presença de sintomas auditivos por meio de um questionário, não sendo utilizado nenhum equipamento. Os sintomas auditivos mais freqüentemente relatados pelos pacientes foram: zumbido $(22,91 \%)$, sensação de ouvido tapado $(18,75 \%)$, tontura $(15,62 \%)$, dor à direita $(13,55 \%)$, sensação de diminuição da audição (11,97\%), vertigem $(9,90 \%)$ e dor à esquerda $(7,30 \%)$. Outras queixas referidas pelos pacientes foram: dor e estalos na ATM $(28,31 \%)$, hábitos parafuncionais $(18,07 \%)$, dor de cabeça $(13,85 \%)$, dor na face e pescoço $(11,44 \%)$, limitação dos movimentos mandibulares $(9,03 \%)$ e dificuldade durante a mastigação $(8,43 \%)$.

No presente estudo, a freqüência da síndrome vestibular periférica nos achados do exame vestibular foi de $74,0 \%$, sendo $40,7 \%$ de síndromes deficitárias e $33,3 \%$ de síndromes irritativas, fato observado pelos autores ${ }^{5,19,25}$. Os procedimentos de avaliação vestibular realizada pelos autores não foram esclarecidos na literatura pesquisada para que fosse possí- 
vel ter um conhecimento de tais procedimentos.

\section{CONCLUSÃO}

As queixas mais freqüentes foram: dificuldade ou dor ao movimento do pescoço, dor irradiada para ombro/braço, zumbido e formigamento de extremidade superior $(77,7 \%)$ em cada, tontura e dor de cabeça $(66,6 \%)$, ansiedade $(55,5 \%)$, sensação de cabeça oca, agitação durante o sono e depressão $(51,8 \%)$ em cada queixa.
A alteração no exame vestibular ocorreu em 74,0\% dos pacientes, na prova calórica e houve freqüência de alteração do sistema vestibular periférico e da síndrome vestibular periférica deficitária. Observou-se, na presente pesquisa, um número elevado de alteração do sistema vestibular em pacientes com DTM. Com isso, ressalta-se a importância da incorporação do exame labiríntico nesse tipo de população, bem como da relevância de pesquisas a respeito da eficácia da reabilitação labiríntica na remissão e/ou superação da sintomatologia nesses pacientes.

\begin{abstract}
Purpose: to evaluate the vestibular functioning in patients with temporomandibular joint dysfunction. Methods: 27 female patients were evaluated, with age varying from 30 to 53 -year-old, referred from the Centre of Diagnosis and Treatment of Temporomandibular Joint Dysfunction to the Otoneurological Laboratory of Tuiuti University of Paraná. The following exams were carried out: anamnesis, otoscopy and vestibular evaluations through vectoelectronystagmography. Results: the most frequent complaints were: difficulty or pain with movement of the neck $(77.7 \%)$, pain irradiated to the shoulder/arm $(77.7 \%)$, tinnitus and paresthesia of superior extremities $(77.7 \%)$ in each one, dizziness and headaches $(66,6 \%)$, anxiety (55.5\%), empty head sensations, agitation during sleep and depression (51.8\%) in each one. The vestibular exam showed alterations in 20 patients $(74.0 \%)$ in the caloric test. There was a prevalence of alterations in the peripheral vestibular system. There was a prevalence of deficient peripheral vestibular syndrome. Conclusion: the importance of studying the relationship between the vestibular system and temporomandibular joint dysfunction is emphasized, since a high number of alterations in the labyrinthic exam have been noted in this research.
\end{abstract}

KEYWORDS: Temporomandibular Joint; Dizziness; Electronystagmography; Vertigo; Labyrinth Diseases

\section{REFERÊNCIAS}

1. Costen JB. A syndrome of ear and sinus symptoms dependent upon disturbed function of the temporomandibular joint. 1934. Ann. Otol. Rhinol. Laryngol. 1997; 106(10):805-19.

2.Dworkin SF, Huggins KH, LeResche L, Van Korff M, Howard J, Truelove E, et al. Epidemiology of signs and symptoms in temporomandibular disorders: clinical signs in cases and controls. J Am Dent Assoc. 1990; 120(3):273-81.

3.Bell WE. Clinical diagnosis of the pain-dysfunction syndrome. J Am Dent Assoc. 1969; 79(1):154-60.

4. Myrhaug $\mathrm{H}$. The incidence of ear symptoms in cases of malocclusion and temporo-mandibular joint disturbances. Br J Oral Surg. 1964; 2(1):28-32.

5.Morgan DH. Dysfunction, pain, tinnitus, vertigo corrected by mandibular joint surgery. J South Calif Dent Assoc. 1971; 39(7):505-34.

6.Williamson $\mathrm{EH}$. The interrelationship of internal derangements of the temporomandibular joint, headache, vertigo and tinnitus: a survey of 25 patients. Facial Orthop Temporomandibular Arthol. 1986; 3(1):13-7.
7.Brown JT, Walker JI. Excessive somatic concern: diagnostic and treatment issues. In: Walker JI, Brown JT, Gallis HA, editors. The complicated medical patient. New York: Human Sciences Press; 1987. p. 13-30.

8.Ash CM, Pinto OF. The TMJ and the middle ear: structural and functional correlates for aural symptoms associated with temporomandibular joint dysfunction. Int J Prosthodont. 1991; 4(1):51-7.

9. Garcia de Hombre AM. Transtorno doloroso y vértigo referido al oído: derivación frecuente al ORL. An Med Interna. 2005; 22(2):88-90.

10. Tuz HH, Onder EM, Kisnisci RS. Prevalence of otologic complaints in patients with temporomandibular disorder. Am J Orthod Dentofacial Orthop. 2003; 123(6):620-3.

11. Manni UM, Brunori P, Giuliani M, Modoni M, Bizzi GI. Sintomi otovestibolari nei pazienti com disfunzioni dell'articolazione temporomandibolare: studio elettromiografico. Minerva Stomatol. 1996; 45(1-2):1-7. 12. Brandt T, Daroff RB. Physical therapy for benign paroxysmal positioning vertigo. Arch Otolaryngol. 1980; 106:484-5.

13. Padovan I, Pansini M. New possibilities of analysis in electronystagmography. Acta Otolaryngol. 1972; 
73(2):121-5.

14. Mangabeira-Albernaz PL, Ganança MM, Pontes PAL. Modelo operacional do aparelho vestibular. In: Mangabeira-Albernaz PL, Ganança MM. Vertigem. 2. ed. São Paulo: Moderna; 1976. p. 29-36.

15. Goodfriend DJ. Abnormalities of the mandibular articulation. J Am Dent Assoc. 1934; 21:204-18.

16. Chole RA, Parker WS. Tinnitus and vertigo in patients with temporomandibular disorder. Arch Otolaryngol Head Neck Surg. 1992; 118(8):817-21.

17. Brookes B, Maw AR, Coleman M. "Costen's syndrome": correlation or coincidence: a review of 45 patients with temporomandibular joint dysfunction, otalgia and other aural symptoms. Clin Otolaryngol. 1980; 5(1):23-36.

18. Erlandsson SI, Rubistein B, Axelsson UM, Carlsson SG. Psichological dimensions in patients with disabling tinnitus anal craniomandibulares disorders. Br Audiol. 1991; 25(1):15-24.

19. Vieira MM, Ávila CRB, Caovilla, HH, Ganança MM. Repercussão otoneurológica da disfunção da articulação têmporo-mandibular. Acta AWHO. 1992;
11(2):68-72.

20. Ren YF, Isberg A. Tinnitus in patients with temporomandibular joint internal derangement. Cranio. 1995; 13(2):75-80.

21. Fanali S, Cerri A. Inquadramento otoneurológico e odontostomatológico della sindrome algico-disfinzionale di Costen. Minerva Stomatol. 1993; 42:37-43.

22. Felício CM, Oliveira JAA, Nunes LJ, Jeronymo $L F$, Jeronymo RR. Alterações auditivas relacionadas ao zumbido nos distúrbios otológicos e da articulação têmporo-mandibular. Rev Bras Otorrinolaringol. 1999; 65(2):141-6.

23. D'Antonio W, Ikino CMY, Castro SM, Balbani APS, Jurado JRP, Bento RF. Distúrbio temporomandibular como causa de otalgia: um estudo clínico. Rev Bras Otorrinolaringol. 2000; 66(1):46-50.

24. Mazzetto MO, Nascimento G, Gomes NM. Estudo da prevalência das alterações das funções estomatognáticas em pacientes com disfunção temporomandibular. J Bras Fonoaudiol. 2002; 7:140-7. 25. Koskinen J, Paavolainen M, Roschier J. Otological manifestations in temporomandibular joint dysfunction. J Oral Rehabil. 1980; 7(3):249-54.

RECEBIDO EM: 17/01/2007

ACEITO EM: 11/04/2007

Endereço p/correspondência:

Rua Gutemberg, n०99 $9^{\circ}$ and.

Curitiba - PR

CEP: $80420-030$

Tel / Fax: (41) 33317807

E-mail: bianca.zeigelboim@utp.br 